\title{
Effect of liquid bridge volume on the instability in small-Prandtl-number half zones
}

\author{
Q.-S. Chen ${ }^{\mathrm{a}, *}$, W.-R. Hu $\mathrm{Hu}^{\mathrm{a}}$, V. Prasad ${ }^{\mathrm{b}}$ \\ anstitute of Mechanics, CAS, Beijing 100080, People's Republic of China \\ ${ }^{\mathrm{b}}$ Consortium for Crystal Growth Research, State University of New York at Stony Brook, NY 11794-2300, USA \\ Received 29 June 1998; accepted 12 January 1999 \\ Communicated by T. Hibiya
}

\begin{abstract}
A perturbation method is used to examine the linear instability of thermocapillary convection in a liquid bridge of floating half-zone filled with a small Prandtl number fluid. The influence of liquid bridge volume on critical Marangoni number and flow features is analyzed. The neutral modes show that the instability is mainly caused by the bulk flow that is driven by the nonuniform thermocapillary forces acting on the free surface. The hydrodynamic instability is dominant in the case of small Prandtl number fluid and the first instability mode is a stationary bifurcation. The azimuthal wave number for the most dangerous mode depends on the liquid bridge volume, and is not always two as in the case of a cylindrical liquid bridge with aspect ratio near 0.6. Its value may be equal to unity when the liquid bridge is relatively slender. (C) 1999 Elsevier Science B.V. All rights reserved.
\end{abstract}

Keywords: Thermocapillary; Oscillations; Instability; Microgravity

\section{Introduction}

Thermocapillary convection in a floating halfzone plays an important role in the case of crystal growth using the floating zone method in a microgravity environment. The oscillatory Marangoni convection in the molten material may induce crystal

\footnotetext{
* Corresponding author. Present address: Department of Mechanical Engineering, State University of New York at Stony Brook, NY 11794-2300, USA. Tel.: + 1-516-632-8339/9793; fax: $+1-516-632-8544$.

E-mail address: qschen@pml.eng.sunysb.edu (Q.-S. Chen)
}

defects such as inhomogeneities and impurity striations during the growth process. Extensive experiments and theoretical analyses have been performed to study the thermocapillary convection in the floating half-zone melt and the associated instabilities [1-8]. Most of the experiments are, however, conducted for large Prandtl number fluids, $\operatorname{Pr} \geqslant 1$. $\operatorname{Pr}$ is defined as $\operatorname{Pr}=v / \alpha$, where $v$ and $\alpha$ denote kinematic viscosity and thermal diffusivity, respectively. Melts of semiconductors, metals, and alloys are liquids of small Prandtl number, which exhibit very different behavior than the large Prandtl number fluids. Only a few experiments, such as those conducted by Cröll et al. [5] 
and Han et al. [9], have been reported on the onset of oscillatory convection in the floating half-zone of small Pr fluid; a critical Marangoni number for the onset of time-dependence is observed to be about 900 in the case of a mercury liquid bridge [9]. Observation of flow and temperature fields in the liquid bridge is generally very difficult in the case of low Pr fluids because of its opaqueness, and only very sophisticated and expensive techniques such as X-ray diagnostics, can provide some information on the velocity field in semiconductor melts [8]. Stability analysis and numerical simulation can therefore be very useful in the study of thermocapillary convection in a floating half-zone melt of small Prandtl number fluids. Such studies can provide a better understanding of transport phenomena in semiconductor materials processing. Furthermore, the materials processing in the microgravity environment needs more quantitative information on hydrodynamic instability and thermocapillary convection.

Rupp et al. [10] and Levenstam and Amberg [11] have performed three-dimensional numerical simulations of thermocapillary convection in a floating half-zone cavity of aspect ratio 0.4 and 0.5 ; the aspect ratio defined as, $A=L / 2 R_{0}$, where $L$ and $R_{0}$ are the height and radius of the liquid bridge, respectively. They found that the most dangerous mode in a cylindrical liquid bridge is a stationary asymmetric state with an azimuthal wave number, $m=2$. Wanschura et al. [12] performed a linear stability analysis of the flow in a cylindrical floating half-zone and confirmed that the most dangerous mode is indeed associated with $m=2$. Savino and Monti [13] conducted transient, threedimensional simulations, and observed that the critical wave number is quite sensitive to the geometric aspect ratio of the bridge. It should be noted that all of the above numerical studies are limited to the cylindrical liquid bridge. In addition, the effect of liquid bridge volume, which is one of the sensitive critical parameters, has been studied only for the gravitational environment and large Prandtl numbers using experiments [14], direct numerical analysis $[15,18]$ and linear stability analysis $[16,17]$.

In the present paper, thermocapillary convection and the associated instabilities in a floating half- zone of small Pr fluid are studied for microgravity environment. It is observed that the instability features are quite different between the small and large Prandtl number fluids, and that the liquid bridge volume is an important parameter.

\section{Mathematical description and numerical method}

A floating half-zone with constant temperatures on both the lower and upper ends as shown in Fig. 1, is considered for the present analysis. Two rods of the same radii $R_{0}$, that hold the liquid bridge, are co-axial and separated by a gap, $L$. The lower and upper end temperatures are assumed to be $T_{L}$ and $T_{L}+\Delta T$, respectively. Only the static deformation of the free surface is considered here and the dynamic deformation of the free surface is neglected.

In addition to Prandtl number, the present problem is governed by the following dimensionless parameters:

$V=\frac{\int_{0}^{L} R^{2} \mathrm{~d} z}{R_{0}^{2} L}, \quad \operatorname{Re}=\frac{U_{0} R_{0}}{v}, \quad \mathrm{Ma}=\frac{\left|\sigma_{\mathrm{T}}^{\prime}\right| \Delta T R_{0}}{\rho v \alpha}$,

$\mathrm{Bi}=\frac{h R_{0}}{k}$,

where $V, \mathrm{Re}, \mathrm{Ma}, \mathrm{Bi}$ represent dimensionless volume of the liquid bridge, Reynolds number, Marangoni number and Biot number, respectively. The constants $\rho, k, h, \sigma_{\mathrm{T}}^{\prime}$ denote the density, thermal conductivity, heat transfer coefficient and surface tension variation with respect to temperature, respectively. The above dimensionless physical quantities are obtained by using the velocity, length and time scales as $U_{0}=\left|\sigma_{T}^{\prime}\right| \Delta T / \rho v, R_{0}$ and $R_{0} / U_{0}$, respectively. The cylindrical polar coordinate system $(r, \theta, z)$ is used for the present analysis as shown in Fig. 1, and the free surface boundary $R(z)$ is considered as a function of $z$.

In the linear stability analysis of thermocapillary convection in the floating half zone, a steady, axisymmetric basic state is obtained through numerical computations. The perturbation quantities of the velocities, $\boldsymbol{u}=(u, v, w)$, pressure, $p$, and temperature, $T$, are imposed to that of the basic state $\boldsymbol{u}_{0}=\left(u_{0}, 0, w_{0}\right), p_{0}$ and $T_{0}$. For example, the 


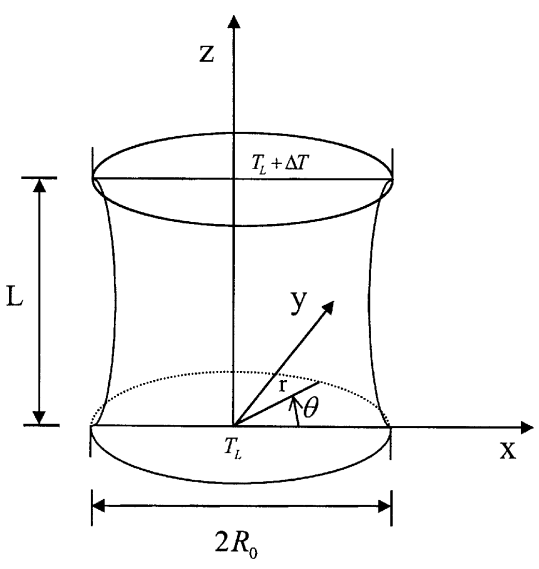

Fig. 1. Schematic of a floating half-zone.

component of radial velocity $U$ may be written as

$U=u_{0}+\varepsilon u$,

where the parameter $\varepsilon$ is very small. The dimensionless governing equations for perturbed quantities in the microgravity environment can then be expressed as follows:

$\frac{\partial \boldsymbol{u}}{\partial t}+\boldsymbol{u} \cdot \nabla \boldsymbol{u}_{0}+\boldsymbol{u}_{0} \cdot \nabla \boldsymbol{u}=-\nabla p+\frac{1}{\mathrm{Re}} \nabla^{2} \boldsymbol{u}$,

$\nabla \boldsymbol{u}=0$,

$\frac{\partial T}{\partial t}+\boldsymbol{u} \cdot \nabla T_{0}+\boldsymbol{u}_{0} \cdot \nabla T=\frac{1}{\operatorname{Re} \operatorname{Pr}} \nabla^{2} T$.

The boundary conditions at the ends $(z=0$ and $z=2 A$ ) are given by

$\boldsymbol{u}=0, \quad \frac{\partial w}{\partial z}=0 \quad$ and $\quad T=0$

while the boundary conditions at the free surface are,

$\boldsymbol{u} \cdot \boldsymbol{n}=0, \quad \boldsymbol{t} \cdot(\boldsymbol{S} \cdot \boldsymbol{n}+\nabla T)=0, \quad \boldsymbol{s} \cdot(\boldsymbol{S} \cdot \boldsymbol{n}+\nabla T)=0$

and $\boldsymbol{n} \cdot \nabla T=-\mathrm{Bi} T$,

where $\boldsymbol{n}$ denotes the normal unit vector at the free surface, and $\boldsymbol{t}$ and $\boldsymbol{s}$ denote the tangential unit vectors in the vertical and horizontal cross sections, respectively, and $\boldsymbol{S}$ denotes the rate-of-strain tensor. In the following analysis, an adiabatic condition is assumed at the free surface, which implies $\mathrm{Bi}=0$.
The conditions at the central axis, $r=0$, are taken as

$u=0, \quad v=0, \quad \frac{\partial w}{\partial r}=0, \quad \frac{\partial T}{\partial r}=0, \quad$ for $m=0$,

$\frac{\partial u}{\partial r}=0, \quad u+\frac{\partial v}{\partial \theta}=0, \quad w=0$

$T=0$, for $m=1$,

$u=0, \quad v=0, \quad w=0, \quad T=0, \quad$ for $m>1$.

The perturbation quantities $(u, v, w, p, T)$ can be expanded as a sum of the spectral terms, for example,

$u=\sum \mathrm{e}^{\sigma t+\mathrm{i} m \theta} u_{m}(r, z)+$ c.c.,

where $\sigma=\sigma_{\mathrm{r}}+\mathrm{i} \sigma_{i}$ with $\sigma_{\mathrm{r}}$ and $\sigma_{i}$ as the increasing rate and frequency of the small perturbations, respectively. In Eq. (2.7), $m$ denotes the azimuthal wave number, $\mathrm{i}$ denotes the complex unit $\sqrt{-1}$, and c.c. denotes the complex conjugate. Eq. (2.7) gives a spectral representation of the perturbation. Algebraic equations can then be obtained by substituting Eq. (2.7) in the governing equations and boundary conditions, and the eigenvalues and eigenfunctions can be determined using the Q-R method as discussed in Ref. [17] for large Pr. For the eigenvalue problem, we have used $19 \times 19$ Chebyshev polynomials in $r$ and $z$ directions, which requires that a generalized eigenvalue equation be solved with a complex matrix of $1444 \times 1444$. Calculations with $21 \times 33$ polynomials in $r$ and $z$ directions were also performed to compare with the results obtained by using $19 \times 19$ polynomials, and the agreement was found to be within $1 \%$. The details of the numerical method can be found in Ref. [17].

To validate the present implementation of the stability analysis, selected results have been obtained for $A=0.5, V=1$ and $\operatorname{Pr}=0.001$ and 0.01 , and compared with those obtained by Chen et al. [7]. The agreement is within $\pm 5 \%$. It can be seen from Table 1, that both solutions predict the azimuthal wave number of two for the most dangerous mode. 
Table 1

Comparison of present results with that obtained by Chen et al. [7]

\begin{tabular}{lll}
\hline Prandtl Number & $\mathrm{Ma}_{\mathrm{cr}}$ (Chen et al.) & $\mathrm{Ma}_{\mathrm{cr}}$ (Present) \\
\hline $\operatorname{Pr}=0.001$ & $1.79(m=2)$ & $1.87(m=2)$ \\
$\operatorname{Pr}=0.01$ & $18.9(m=2)$ & $19.8(m=2)$ \\
\hline
\end{tabular}

\section{Numerical results}

The flow pattern for basic state of convection is shown in Fig. 2 for $A=0.6, V=0.8, \mathrm{Pr}=0.001$, and $\mathrm{Re}=2000$. Since the flow pattern for basic state, for a fixed Reynolds number, show only a small difference in the Prandtl number range of $\operatorname{Pr}=0.001-0.1$, results for only $\operatorname{Pr}=0.001$ are shown here. However, the temperature distributions in the basic state strongly depend on Prandtl number, as shown in Fig. 3A-Fig. 3C for Prandtl number of $0.001,0.01$ and 0.1 , respectively. The temperature profiles in the bulk region approach nearly linear distribution in the case of very small Prandtl number such as $\operatorname{Pr}=0.001$ (Fig. 3A), except for the corner region when $V / V_{0} \neq 1$. But, they start deviating from the linear profile as $\operatorname{Pr}$ is increased and a significant variation is seen in Fig. $3 \mathrm{C}$ when $\mathrm{Pr}=0.1$. In this case, there exist relatively large temperature gradients near the cold corner of the liquid bridge.

The critical Marangoni number, $\mathrm{Ma}_{\mathrm{cr}}$, as a function of the liquid bridge volume, are shown in Fig. 4A-Fig. 4C for $A=0.6$ and Prandtl number of $0.001,0.01$ and 0.1 , respectively. Since the melt of many semiconductor materials have Prandtl number, $\operatorname{Pr}=O\left(10^{-2}\right)$, critical curves for $\operatorname{Pr}=0.01$ are shown in more detail in Fig. 4B, for azimuthal wave number of $0,1,2$ and 3 . The results presented here show that the most dangerous mode is $m=2$ when the dimensionless liquid bridge volume, $V>0.8$, and is $m=1$ when $V \leqslant 0.8$. In the case of small Prandtl number $(\mathrm{Pr} \leqslant 0.1)$, it is evident that the most dangerous mode is $m=2$ for a relatively thick liquid bridge, for which the cylinder is a special case where there is no concavity. For a relatively slender liquid bridge the most dangerous mode is obtained as $m=1$. It should be noted that, the wavenumber

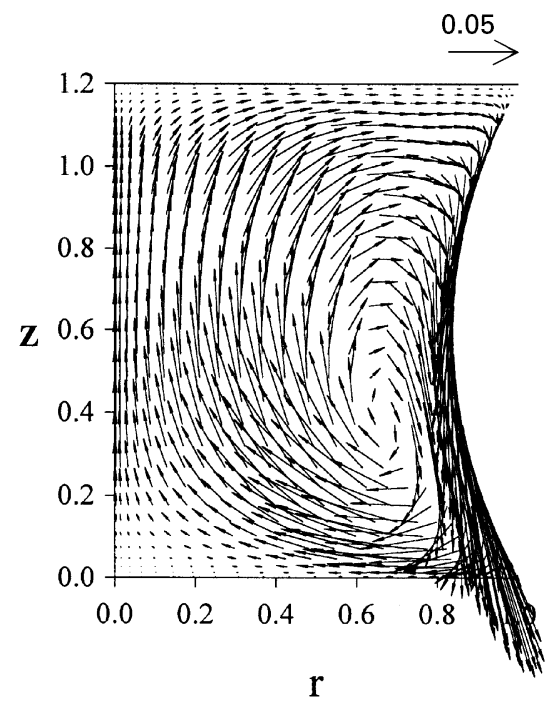

Fig. 2. Basic flow patterns in a liquid bridge of $A=0.6, V=0.8$, $\operatorname{Pr}=0.001$ and $\operatorname{Re}=2000$.

of the most unstable mode, $m=1$, does not change in a large range of liquid bridge volume in the case of larger Prandtl number fluids $(\operatorname{Pr} \geqslant 1)$ [17]. The liquid bridge volume is also a sensitive parameter in the case of small Prandtl numbers. The critical Marangoni number in the case of a smaller liquid bridge volume may be several times larger than that for the large volumes. The critical Marangoni number curve as a function of the liquid bridge volume for small Prandtl number fluids is quite different from that of the large Pr fluids [15,17].

The perturbed temperature and flow fields in a liquid bridge of $A=0.6, V=0.8, \mathrm{Pr}=0.01$ and $\mathrm{Ma}=20$ are shown in Fig. 5 for the most unstable mode, $m=1$. The left figures $\mathrm{A}$ and $\mathrm{C}$ are associated with the perturbed temperature fields in the horizontal cross-section, $z=0.6$, and the vertical cross section $y=0$, respectively. The right figures $\mathrm{B}$ and $\mathrm{D}$ on the other hand represent the corresponding perturbed flow fields. The perturbed flow pattern in Fig. 5B is symmetric with respect to $y=0$, and there are two cells including one small degenerated cell near the lower corner, as shown in Fig. 5D. Fig. 5B shows that the azimuthal velocity changes periodically and the fluid does not rotate as an entity, since the imaginary part of the growth 


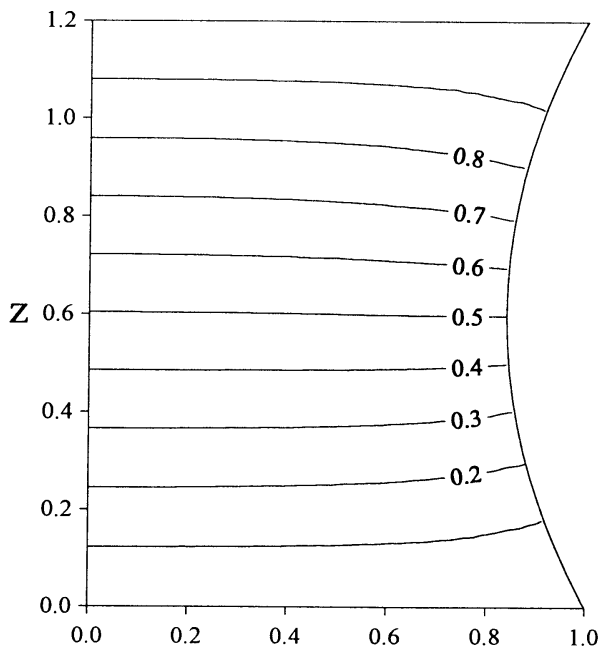

(A) r

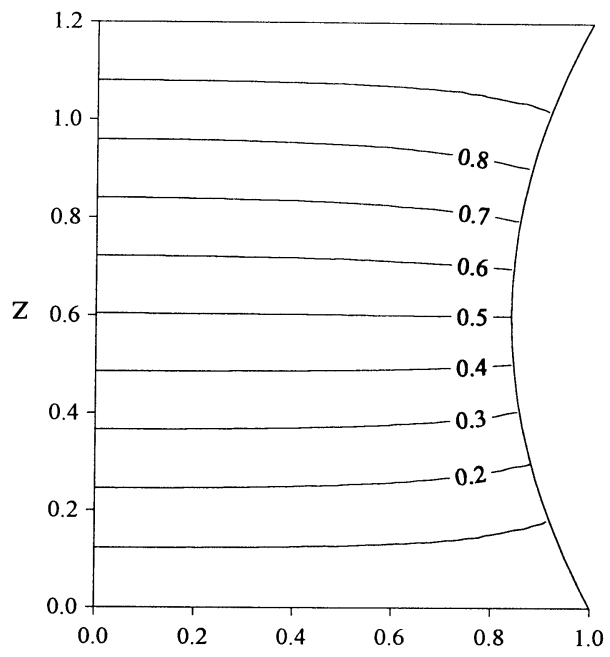

(B) r

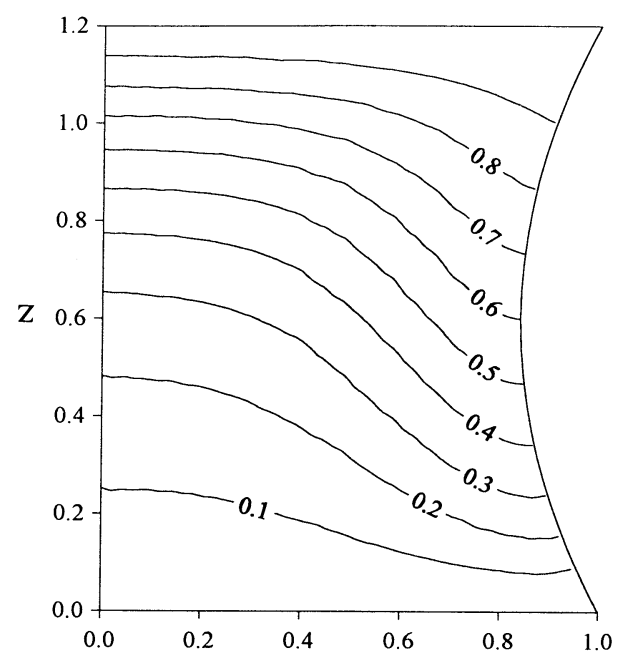

(C)

r

Fig. 3. Basic temperature distributions in a liquid bridge of $A=0.6, V=0.8$, and $\operatorname{Re}=2000$ where $\operatorname{Pr}=0.001(\mathrm{~A}), \operatorname{Pr}=0.01(\mathrm{~B})$, and $\operatorname{Pr}=0.1(\mathrm{C})$.

rate is zero. The perturbed temperature in the vertical cross section has a minimum in the plane, $\theta=0$ and a maximum in the plane, $\theta=\pi$, and the perturbed temperature distribution is symmetric to the vertical plane, $y=0$.

The critical Marangoni number, $\mathrm{Ma}_{\mathrm{cr}}$, as a function of Prandtl number for $A=0.6$ and $V=0.8,1$ and 1.2 are given in Fig. 6 for the most unstable mode. The instability is mainly hydrodynamic, the first bifurcation being stationary for $\operatorname{Pr} \leqslant 0.1$. The perturbed state will increase exponentially until it reaches a stationary state. The instability in the case of $\operatorname{Pr} \geqslant 1$ is induced through the coupling between the hydrodynamic and thermocapillary effects, which has been discussed in Ref. [17] and the frequency is not zero. 


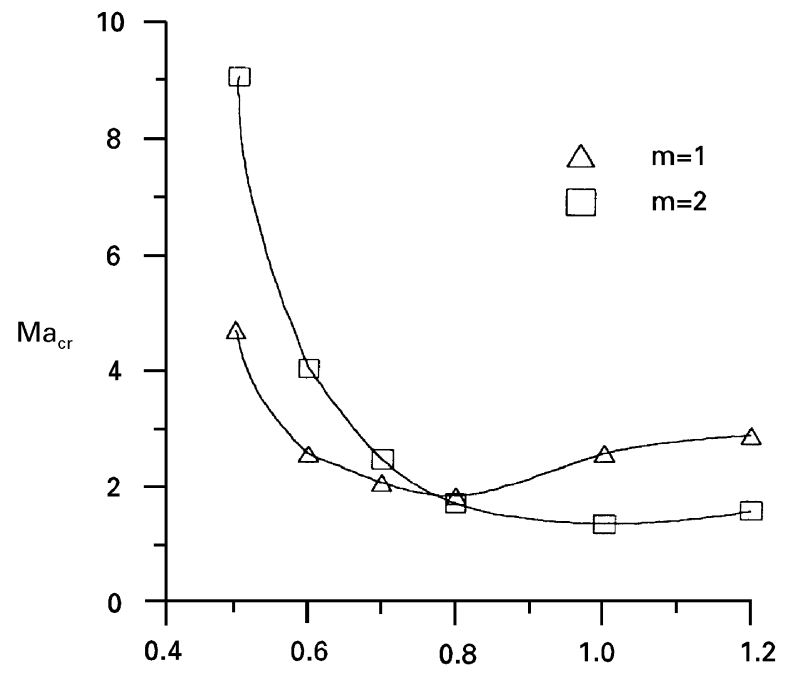

(A)

V

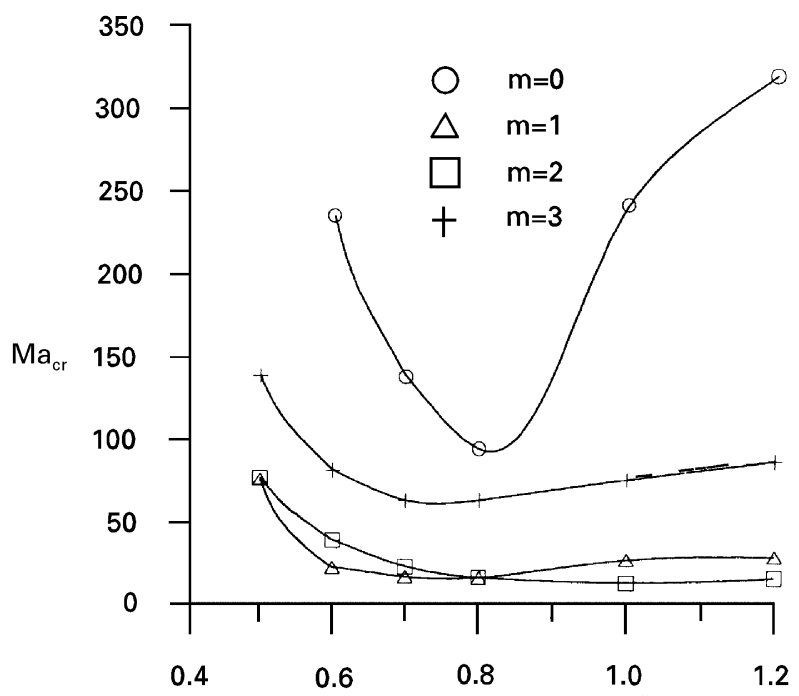

(B)

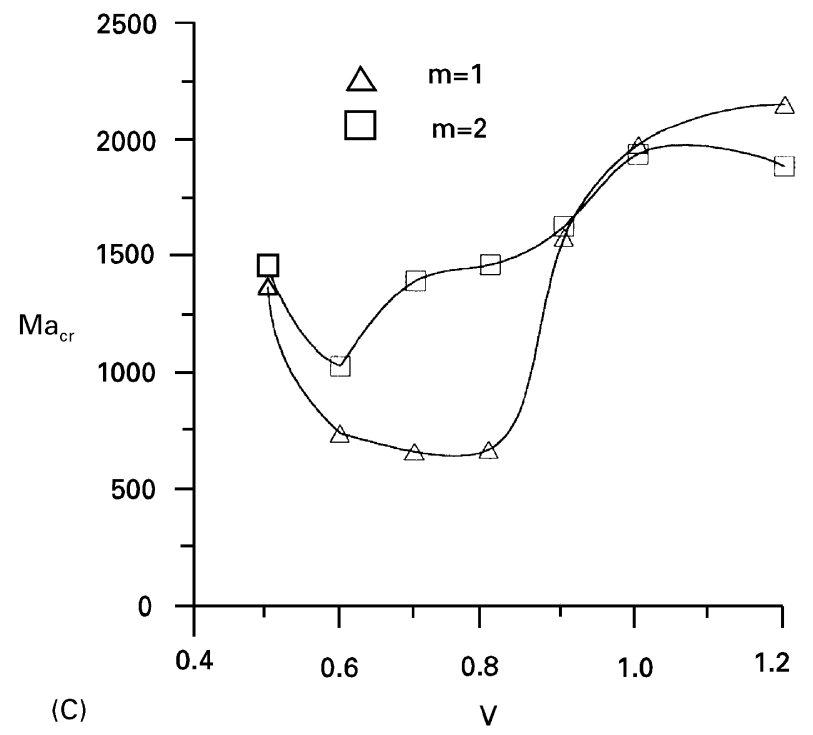

Fig. 4. Critical Marangoni number as a function of the liquid bridge volume with $A=0.6$, and $\operatorname{Pr}=0.001$ (A), $\operatorname{Pr}=0.01$ (B), and $\operatorname{Pr}=0.1(\mathrm{C})$.

\section{Discussions and conclusion}

In the present work, the perturbation analysis is applied successfully to study the instability of thermocapillary convection in a floating half-zone cavity of low Prandtl number fluids, and the basic state of steady and symmetric convection is calculated using the spectral method [16]. The influence of liquid bridge volume on instability has been discussed in detail. The cylindrical liquid bridge, which has been investigated in more details in the past, is a special case of the present analysis. However, the critical Marangoni number obtained from the stability analysis and that predicted by the 

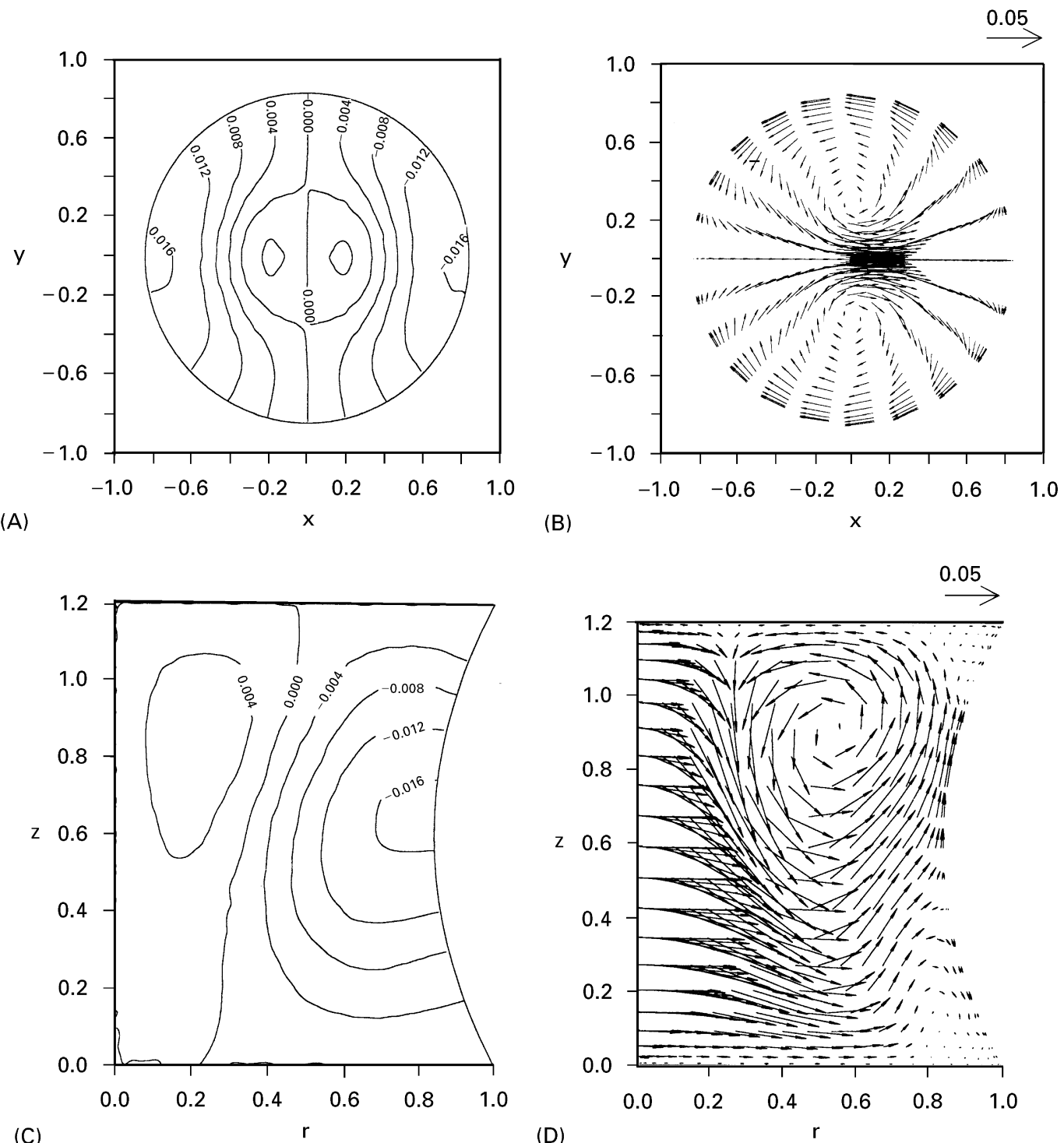

Fig. 5. Perturbed temperature distribution and flow field in a liquid bridge of $A=0.6, V=0.8, \operatorname{Pr}=0.01$ and $\mathrm{Ma}=20$ for the most unstable mode, $m=1$. The upper (A and B) diagrams show the temperature and velocity distributions in the horizontal cross section at $z=0.6$ and the lower $(\mathrm{C}$ and $\mathrm{D})$ diagrams present them in the vertical cross section $\theta=0$.

numerical model in Ref. [13] are much smaller than that measured experimentally [9]. Both the stability analysis and numerical simulation predict the onset of asymmetric convection from steady, symmetric state, while the experimental measurements show the onset of oscillatory convection from the asymmetric convective state. The critical Maran- goni number predicted by the former methods is lower than that obtained experimentally [11].

The instability is found to be mainly hydrodynamic [12] and the first bifurcation is stationary for $\operatorname{Pr} \leqslant 0.1$. The most dangerous mode is $m=1$ for a relatively slender liquid bridge and $m=2$ for a relatively fat liquid bridge, which also 


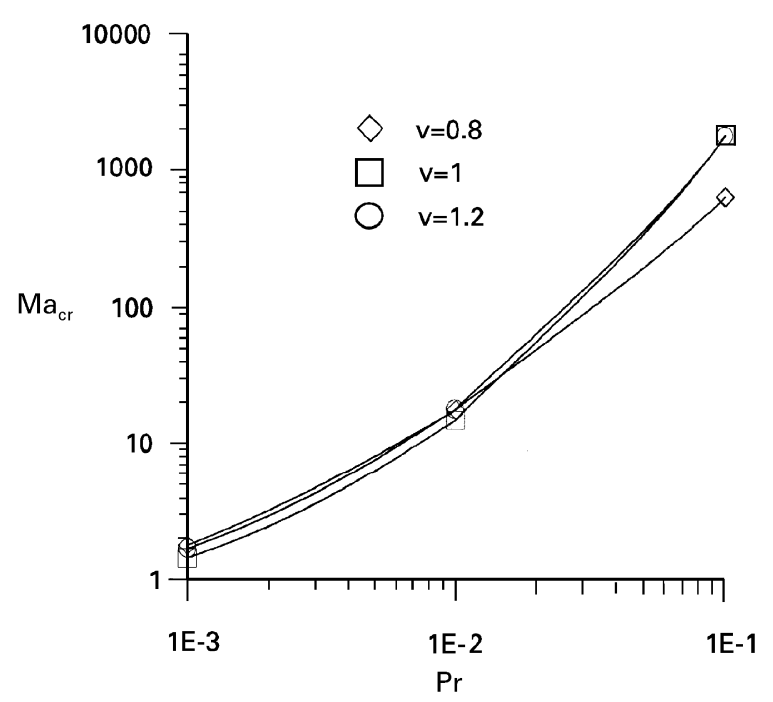

Fig. 6. Critical Marangoni number $\mathrm{Ma}_{\mathrm{cr}}$ (A) as a function of Prandtl number in a liquid bridge of $A=0.6$ and $V=0.8,1$ and 1.2 , for the most unstable mode $m=2$, except for the cases of $V=0.8$ and $\operatorname{Pr}=0.01$ and 0.1 which show the most unstable mode $m=1$.

depend on the Prandtl number. Salient features of different liquid bridge volumes are shown and it is demonstrated that the critical Marangoni number may change significantly in a range of Prandtl number and aspect ratio. In addition, the liquid bridge volume is a sensitive critical parameter for the onset of asymmetric or oscillatory convection. The stability analysis can only determine the stability boundary and not the behavior beyond the threshold. The complex evolution process can only be predicted by a transient, three-dimensional model.

\section{Acknowledgements}

The authors are thankful to G. Chen for helpful discussions and calculations of critical Marangoni number to compare with our results.

\section{References}

[1] C.E. Chang, W.R. Wilcox, J. Crystal Growth 28 (1975) 8.

[2] C.H. Chun, W. Wuest, Acta Astronaut. 5 (1978) 681.

[3] D. Schwabe, A. Scharmann, J. Crystal Growth 46 (1979) 125.

[4] Y. Kamotani, S. Ostrach, M. Vargas, J. Crystal Growth 66 (1984) 83.

[5] A. Cröll, W. Müller-Sebert, R. Nitsche, Mater. Res. Bull. 24 (1989) 995.

[6] W.R. Hu, H.T. You, C.H. Cao, Sci. China A 4 (1992) 383.

[7] G. Chen, A. Lizée, B. Roux, J. Crystal Growth 180 (1997) 638.

[8] S. Nakamura, T. Hibiya, K. Kakimoto, N. Imaishi, S. Nishizawa, A. Hirata, K. Mukai, S. Yoda, T.S. Morita, J. Crystal Growth 186 (1998) 85.

[9] J.H. Han, Z.W. Sun, L.R. Dai, J.C. Xie, W.R. Hu, J. Crystal Growth 169 (1996) 129.

[10] R. Rupp, G. Müller, G. Neumann, J. Crystal Growth 97 (1989) 34.

[11] M. Levenstam, G. Amberg, J. Fluid Mech. 297 (1995) 357.

[12] M. Wanschura, V.M. Shevtsova, H.C. Kuhlmann, H.J. Rath, Phys. Fluid 7 (1995) 912.

[13] R. Savino, R. Monti, Appl. Sci. Res. 56 (1996) 19.

[14] W.R. Hu, J.Z. Shu, R. Zhou, Z.M. Tang, J. Crystal Growth 142 (1994) 379.

[15] Z.M. Tang, W.R. Hu, J. Crystal Growth 142 (1994) 385.

[16] Q.S. Chen, W.R. Hu, Int. J. Heat Mass Transfer 40 (1997) 757.

[17] Q.S. Chen, W.R. Hu, Int. J. Heat Mass Transfer 41 (1998) 825 .

[18] Z.M. Tang, W.R. Hu, J. Crystal Growth 192 (1998) 335. 